\title{
ZU OTFRID.
}

\section{Otfrids accente.}

Die accente in Otfrids Evangelienbuch sind bisher meist nur in bezug auf die sie tragenden silben beachtet worden; ihre bedeutung im verse ist eingehend noch nicht erörtert. Einzelne betrachtungen in dieser hinsicht habe ich in der einleitung zu meiner Otfridausgabe gegeben; doch versprach ich daselbst, ebenso wie in der Litteraturgeschichte und Grammatik s. 467, an anderem orte eingehendere untersuchungen zu liefern. Diesem versprechen komme ich hiermit nach.

Otfrid war, als er es unternahm, den gereimten vers der lateinischen hymnen fur eine grössere, deutsche dichtung zu verwenden, noch lange nicht so sehr aus dem banne der volkstilmlichen dichtungsweise herausgetreten, dass er sich ganz der dieser eigentumlichen recitationsweise hătte entziehen kōnnen. Die im vortrage des allitterierenden verses stark markierten stäbe hob auch er beim lesen des seinigen hervor: die bindung durch allitteration fiel bei ihm freilich hinweg, aber des hilfsnittels, welches die betonung der stabsilbon in der langzeile für die declamation darbot, woll te er sich nicht begeben, weil der ersatz der rhythmischen betonung, den der vers der hymnen bot, deutschen ohren noch $\mathrm{zu}$ fremd war, noch konnte er es, weil der reim ihm bereits schwicrigkeiten genug machte und die dichterische sprache der zeit in ihren copulativen und adversativen fugungen gerade fur cine derartige betonungsweise vorbereitet war. Endlich durfte er es auch nicht, wenn er seiner dichtung nicht jeden boden im volke entziehen wollte. 
Die accente stehen an den stellen, wo wir in den allitterierenden langzeilen die stäbe $\mathrm{zu}$ suchen haben, und ihr gesetz muss mit dem der stabreimenden dichtung ubereinstimmen. Lässt sich das erweisen, so haben wir in Otfrids accenten eine reichbaltige quelle mehr furr die kenntnis der regeln des stabreims, und andererseits in den gesetzen des stabreimenden verses einen willkonmenen anhalt fur die stellen, an denen die hss. zweifel übrig lassen, welcher der accente der von Otfrid zuletzt für richtig gehaltene sei.1) Ich gehe in meinen untersuchungen von den halbversen aus, in denen $\mathrm{V}$ und $P$ tibereinstimmen. Wenn darnach die stellen in $P$, in denen es abweicht, sich als besserungen herausstellen, so wcrden dieselben ein neuer beweis dafür seiu, dass $P$ den authentischen text bietet.

In gange meiner untersuchungen knüpfe ich an das in der einl. meiner ausgabe s. 149-171 gesagte an und folge besonders den untersuchungen $M$. Riegers über den allitterierenden vers, nebst deren ergänzungen durch Sievers, Horn, Ries.

Es liegt ja in der natur jeder betonung, das gleichwertige durch gleichen ton hervorzuheben, und dieses grundgesetz ist auch von bedeutung fur die allitterierende dichtung geworden, insofern es in dieser die bildung copulativer und adversativer begriffsvereinigungen, besonders im ersten halbverse, beförderte. Von ungleichartigem erhält ebenso naturgemäss das wichtigere den ton. Diese beiden grundsätze bewahrheiten sich in den gesetzen der stabreimenden dichtung, und wo das nicht der fall \%u sein scheint, da war eben der begriff vom wichtigeren und dem weniger wichtigen bei unseren dichtenden vorfahron ein anderer.

Geht einem substantivum cin anderes in genetiv voran, so hat in der regel das letztere den ton. ${ }^{2}$ ) Die fälle sind sehr häufig, so ther gótes sun, ther gótes geist, gótes boto, gótes man, thaz gótes hus, gótes uuihi, thiu gótes krafı, gótes

1) In den citaten lasse ich ohne weitere bemerkung die etwa zu delierenden accente gleich weg.

2) Die fälle, wo auch das zweite wort einen ton trïgt, des verses wegen, kommen hier natürlich nicht in betracht, da sie uns das gesetz nicht veranschaulichen können. 
kind, gótes uuillo, gótes thegana, themo gotes munde II, 4, 50, gótes kornon I, 28, 10, themo gótes biete II, 18, 20, gótes fraga II, 6, 41, gótes minna IV, 5, 47; ähnlich drühtines sun, druihtines lus, druiltines uuillon, thes ménnisgen suil, thes geistes giburt (II, 12, 47); ferner suntono mahtin II, 24, 22, libes frist II, 3, 28, $z i$ then kristes góumon III, 7, 70, und in viclen anderen fällen. Dengemäss verbessert $P$, teils gleich bei der crsten niederschreibung, der a) bereits eine correctur in V vorlag, oder glcichzeitig erfolgte, oder auch b) ohne correctur in $\mathrm{V}$, teils c) bei der spätcren ubercorrectur: a) thero drúhtines unorto II, 14, 73; gótes hus II, 3, 24; gótes sune II, 4, 71; thes crüces hornon II, 9, 83; sámbazdages fra III, 4, 33; thero núhistono guati III, 15, 16; thes kruzes segonon $\mathrm{V}, 2,1$; thes kruizes bouma $\mathrm{V}, 2,8$; thero óstorono fira V, 4, 7 (die stellen III, 14, 43. 72. 18, 60. IV, 5, 42. 44. 6, 9. 23, 21. V, 4, 1 fallen, als im ersten balbverse befindlich, viclleicht weniger ins gewicht, da man dort das weglassen des zweiten accentes, zum teil mit recht, als nachlässigkeit erklären kanu); b) múnnes lust $1,1,17$; gótes sun I, 25, 9; gótes gift II, 14, 23; kristes uuorles V, 23, 47; kristes muas III, 7, 73. 79; kristes muater V, 12, 19; thes liobes thingon V, 23, 42; thiu gótes uuerk V, 12, 51; thes stéines burdin $\mathrm{V}, 4,16$; c) gótes ensi $\mathrm{I}, 15,18$; thes fáter namon $\mathrm{I}, 9,17$ (II, 18, 7 ist in $\mathrm{P}$ eine irrung; der zweite accent ist ungiltig); then gótes druit II, 4, 63; kristes tódes IV, 1, 2. Der gemeinsamen ibercorrectur beider hs8. wcise ich III, 14, 7 thes héresten dóhter zu. Betrachten wir noben dicsen mit leichtigkeit \%u vermelıreuden fällen der von vornherein beobachteten oder durch correctur befolgten regel dic auftretenden abweichungen, so fällt auf, dass dio nucisten der letzteren in dem zweiten balbverse stehen. In diesem setzte, wie wir unten sehen werden (vgl. auch Einl. s. 165, Gramm. s. 467), Otfrid meist éinen accent, und diesen weler ganz an den anfang, noch ganz an den schluss, sondern nüglichst in die mitte, damit er das gewicht des halbverses trilge. Er greht in diesem punkte $\mathfrak{u b e r}$ die uns bekannten regeln des allittericrenden verses hinaus. Hierher gehören folgende stellen in PV: mannes lichamon nam II, 8, 59; gotes druttheganon I, 28, 11; gotes ábulgi II, 13, 38; gotes thionoste I, 16, 9; gotes unört scoumon I, 13,4; thes kindes fáter uuari I, 15, 23; gotes hind heizent 11, 16, 26; dales ébonoti 1, 23, 24 
(vgl. aber I, 9, 36) ferner I, 1, 34. 116. 3, 2. 4, 59. 67. 3,15. 4, 23. 10, 28. 11, 38. II, 2, 26. 4, 39. 6, 55. 9, 12. 12, 13. IV, 17, 14 . 20, 17. 29, 29. V, 6, 38. 22, 1.9. Demgemäss sind folgende correcturen in $\mathrm{P}$ auch gerechtfertigt, resp. erklärt: $1,16,26$. IV, 8, 12. 37, 2. 17. Seltener hatte diese rücksicht auf das gleichgewicht des verses auch im ersten halbverse statt, so in dales ébonoti I, 9, 36; uuega uuólkono I, 5, 6; in sunnun ánaliche III, 13, 42 und in der verbesserung von $\mathrm{P}$ : gibetes ántfangi $\mathrm{I}, 4,73$ und $\mathrm{V}, 4,9$. Durch den gegensatz ist die abweichung von der grundregel der stäbe veranlasst und erklärt in horn héiles nales féhtannes $\mathrm{I}, 10,15$; in kindes inbrusti zi gotes ánahusti I, 4, 42; ebenso in der corr. von P: V, 1, 19 (hórn opp. árma ioh hénti). Bei der grossen menge regelrechter accente sind die folgenden wenigen abweichungen als fluchtigkeiten leicht zu entschuldigen und einfach zu verbessern: I, 16, 7 gotes hús; III, 26, 6 thia gotes dát; 19, 38 thero manno fráuili (wenn in dem letzten beispiele nicht das gefülul einer antithese bei dem dichter als motiv gelten soll). Bei der correctur in P I, 16, 27 mag die absicht einer besonderen hervorhebung vorgelegen haben.

Steht der genetiv nach, so hat das regierende wort den accent, daher die correctur in $P I, 16,16$. Gewichtsausgleichung im zweiten halbverse bedingt eine abweichung in thaz herza fórdrono I, 4, 41 und in der corr. P sluzila himiles III, 12, 37; im ersten halbvers stimma ruafentes I, 23, 19, thaz herza iúdeono $\mathrm{V}, \mathbf{6 , 3 0}$.

Auch für den superlativ, mag er dem genetiv vorangehen oder folgen, gilt die regel, dass das erste wort den ton hat (dor seltenheit der bcispiele wegen fuhre ich auch solche an, wo nuch las zweite wort cinen accent trägt): fúrista thero guati I, 3, 22; fúristo thero liuto II, 12, 2; then fúriston therera uuórolti IV, 12, 63; fúrist ist alles guates III, 24, 57; fürista alles uuihes I, 6, 8; uuihes liobosta II, 11, 45; scázzo diuroston IV, 35, 41. Durch gewichtsausgleichung im zweiten halbverse ist erklärt manno éristo I, 3, 5; manno liobosia 22, 43; barno bézisla 13,10; gote zéizasto 5,16 ; dages liohtosta IV, 33, 10 (vgl. dages héizista II, 14, 10); dreso diurista II, 15, 20.

Attributive verbindung zweier substantiva in demselben halbverse kommt selten vor; doch corrigiert $P$ richtig gegen V: 
druihtin krist I, 26, 2. 1I, 7, 35 (vgl. 1, 12, 14. 11, 2, 16); kriste themo unirle II, 10, 18. Die abweichung gole héilante I, 7, 6 ist durch dic gewichtsausgleich!ng im zweiten halbverse erklärt, bei IV, 20, 2 ther biscof kaiphas uuas thar ioh ther herizoho in uuar scheint der eigenname die ausuahme zu bedingen, und in I, 20, 1 dürfte demnach der accent auf bifínd zu streichen sein, so dass also heród nachträglich einen solchen erhalten hätto (vgl. I, 21, 1).

Bei attributiver verbindung von substantiv und adjectiv hat auch das erste von beiden den ton, z. b. grózara angusti II, 4,36; gúate man II, 7, 16; ebenso nachstehend: fáter stummon I, 9, 23; fon /örasagon luggen II, 23, 8; scáchara urmare IV, 27, 3; héreron thie uuise III, 10, 39 und oft. Demgemäss ist von $\mathrm{P}$ richtig verbessert I, 1, 44 scóni uers (vgl. aber v. 48, wo der fehler noch stehen geblieben); 2, 2 eigan thiu (18,2 eigan línt gewichtsausgleichung im zweiten halbvers); 3,4 míhilo uuinni; 12, 24 guates uuillen; 6, 5 uuih dohter; II, 12, 49 ther guato man; 15, 14 óugon bliden; 14,74 diafemo antuuurle; 8, 51 then gúaton uuin; 9, 2 géisilichero uuorto; 24, 24 ábahen githankon; III, 2, 34 then liobon drost; 2, 37 ther küning irdisgo (vgl. IV, 27, 9); 6, 13. 14. 22. 7, 84. 11, 25. 13, 31. 14, 88. 18, 12. 37. 19, 10. 20, 22. 24, 97 (zugleich corr. in V). 107 (vgl. IV, 6, 18). IV, 5, 41. 55. 62. 7, 26. 11, 52. 12, 55. 20, 24. 22, 18. 29, 13. 37,30 . 38. V, 4, 60. 6, 35. 20, 28. 23, 84. 180. Auch in IV, 19, 43 ther fúristo éuuarto bessert $P$ das richtige ein, während $e 8$ in demselben ausdrucke III, 24, 108 den accent vergisst (gegon V). Folgende abweichungen erklären sich durch gewichtsausgleichung im zweiten halbverse: I, 3, 46 uuorton frénkisgen; 18, 38 unaro kárilati; 17, 40 mihilo únkusti; 18, 39 scono füriburti; 12, 10 so /ronisg irunti; 5, 4 diuri arunti; 3, 34. 37. 5, 21. 56. 15, 14. 17, 40. 65. II, 4, 8. 7, 55. 16, 21. IV, 23, 39. $\mathrm{V}, 20,82$; besonders lehrreich ist III, 26, 22 scónes riches thes hohen himilriches; im ersten balbverse: I, 5, 52 alten sátanasun; 28, 13 hoho guallichi; 3, 25 hohun áltfatera; II, 8, 42. 24, 80. 20, 70. IV, 23, 40; demgemäss in $P$ gebessert: $I, 12,20$ kind níuiboranaz; III, 1, 5 zeichan séltsanu. Dieselben regeln gelten von den zahlen: I, 3, 36 éinlif stunton; 5, 2 thria stunta; 14, 12 fiarzug dago; III, 14, 3 thie scriptora fiari $\mathbf{u}$. s. w. und $\mathbf{P}$ corrigiert richtig: III, 13,43 áhto dagon; 14, 85 zuélif thegana; 
IV, 6, 3 fiar naht; V, 1, 32 fiar hálbun. Dic folgende geringe zahl wirklicher abweichungen von der regel sind als schreibfehler aufzufassen: III, 6, 10 unorolt mihil; V, 15, 17 druhtin griato (in diesen zwei fälen mag der rhetorische ton von einfluss gewesen sein); ferner die fälle, wo $P$ gegen $V$ unrichtiges bietet I, 8, 14 (vgl. v. 13; der acc. ist vergessen); 7, 10 (máru opp. irmeru); III, 20, 7. IV, 8, 23. 23, 43. (vgl. II, 18, 6) 28,19 . Die letzten vier fälle sind schreibfehler und zu verbessern.

Bei prädikativer zusammenstellung zweier nomina hat auch das erste den ton. Die beispiele, namentlich mit der copula $\sin$, sind zahlreich (vgl. besonders die scligpreisungen in II, 16); vgl. auch I, I, 8 iz dúnkal eigun fúntan. Demgemäss corrigiert $\mathrm{P}$ mit recht: I, 4, 9 Unbera uuas thiu quéna; II, 9, 75 gót ther fáter uuari; I, 4,32 sih unórolt méndenti. Die abweichung 1, 27, 1 erklürt sich durch gewichtsausgleichung; ein schreibfehler ist die accentuation von $P$ in $I, 3,16$.

Wenn zwei substantiva, die verschiedene funktionen im selben satze haben, zusammentreffen, so hat das erste den ton; so corrigiert P richtig: I, 4,85 thiu quéna sun uuas drágenti; III, 8, 13 ther unint thaz scif fuar iágonti; wenn es II, 18,11 gegen $\mathrm{V}$ schreibt thaz man mán ni sluagi, so ist das erste man pronominal $\mathrm{zu}$ fassen und der erste ictus auf thaz zu legen. In I, 12, 23 si gote guallichi und in II, 23, 14 in hiafon figun thanne ist wider gewichtsausgleichung. Unrichtig ist die betonung $\mathrm{V}, 6,17$.

Tritt ein adjectiv mit abhängigem casus zusammen, so hat das orsto die nächste berechtigung zum accent (z. b. I, 4, 33 góle filu liuber; II, 18, 24 góte filu léidaz). Demgemäss corrigiort P mit recht: II, 7, 36 muates lind; 9, 4 góumono ádeilon. Durch gewichtsausgleichung sind erklärt: I, 4, 50 kindes ùrminnu; II, 16, 5 muates mámmunte; I, 14, 6 thes kindes háft uuurli; 12, 24 thie fol sin guates unillen. In P I, 4, 5 gote filu druidiu ist ein accent vergessen. Dieselbe regel gilt ubbrigens, wenn der casus von einer präposition regiert wird (I, 1,64 zi uuáfane snélle).

Bei drei nominibus gilt das von Rieger (s.21) aufgestellte gesetz, dass dasjenige an zweiter oder dritter stelle stehende wort, welches $\mathrm{zu}$ dem vorhergehenden in grammatischem rek- 
tionsverhältnis steht, zu diesem auch in enklise des tones steht, mithin keineu ton tragen kann.

Fur ein substantivum, das einen genetiv und cin adjektiv bei sich hat, finden sich bei Otfrid. folgende combinationen:

a) In V, 15, 29 ist die frthere betonung von VP richtiger: thera thikun góles fraga; der reim mag hier von einfluss gewesen sein. Gewichtsausgleichung fand statt in: III, 7, 51 Ihie hohun gótes thegana; IV, 34, 9 manag gótes drut; V, 3, 18 ioh allen gótes theganon. Mit recht aber corrigiert $\mathrm{P}$ in III, 12, 24 ther fúristo drúhtines drut.

b) Durch gewichtsausgleichung im zweiten halbverse erklären sich: I, 14, 21 uuibes érisıa kind; II, 3, 26. 12, 85 ther gotes éinigo sun. Da wegen der eigenartigkeit von Otfrids einrichtung les ersten halbverses passende beispiele nur selten vorkommen, so lässt sich hier nicht absehen, ob 0 . wirklich diese regel noch übte. IV, 26,3 thes liutes mihil ménigi und IV, 16, 18 mánno mihil menigi scheinen zu widersprechen, cbenso die correctur von $\mathrm{P}$ in IV, 5, 39 theist frides furista gisiht.

c) Richtig betont ist III, 24, 36 gótes sun gizámi und auch richtig von $\mathrm{P}$ corr. $\mathrm{I}, 4,21$ gotes boton scónan; I, 25, 9 ther gótes sun selbo; III, 6, 26 ther kristes thegan guato. Gowichtsausgleich im zweiten balbverse hatte statt III, 13, 41. 16, 3. $\mathrm{V}, 17,16$ ther gotes sun frono (dasselbe IV, 19, $51 \mathrm{im}$ ersten halbverse); V, 12, 28 ther gotes sün guater; und demgemäss in $P$ corrigiert: I, 5, 46 gotes sún frono; II, 7, 7 gotes drüt ther maro; 7, 2.1 gotes thégan guater; in III, 4, 11 Engil gotes guato lat $\mathrm{P}$ nur toilweise dic falsche accentuierung von $\mathrm{V}$ berichtigt.

d) Gewichtsausgleichung stellt $P$ her in III, 6, 4 finf thisonton mannes (vgl. v. 53, wo wol auch der zweite accent zu tilgon).

Dasselbe gesetz sollte gelten, wenu von dem cinon genetive noch ein anderer abhïngt, doch hier sehen wir ganz deutlich das streben nach einer responsion des accentes der ersten mit einem auf der dritten silbe hervortreten, auf das ich schon in der einl. zu Otfrid aufmerksam machte und das vielleicht auch in den eben besprochenen fällen sich schon geltend macht: I, 1, 47 in gótes gibotes suiazi; $111,22,1$ thes gótes huses uuihi; 
23, 21 thes gótes sunes máhti. Im zweiten halbverse tritt auch hier gewichtsausgleichung ein: I, 12,6 thes gotes bólen uuorto; III, 7, 25 gerstun kórnes hut; IV, 27, 1 thero uuibo klága gouma; einmal im ersten halbverse: I, 15, 8 thiu uuihi góles geistes.

Substantiv mit genetiv, zu dem ein adjectiv gehört: a) Richtig ist noch $\mathrm{P} V, 23,226$ álles mines libes frist, aber statt des alten gesetzes ist schon responsion des accentes eingetreten im zweiten halbverse: I, 22, 37 thes iungen kindes uuórto; II, 15, 9 siechero manno ménigi; gewichtsausgleichung in zweiten halbverse: I, 12, 27 arges uuillen gilust; III, 23, 33 zuelif dágo ziti; IV, 33, 8 thrio dáges ziti; im ersten halbverse $V, 4,32$ in uuizes snéuuen farauni; demgemäss von $P$ corr. im zweiten halbverse I, 6, 8 uuahsmo réues thines; im ersten: IV, 20, 11 thes argen uuillen herti; 23, 22 alten nides uuillen. Auffällig ist III, 7, 23 thes sarphen uuizodes not. b) $P$ corrigiert richtig III, 6, 54 séti sibun broto.

In einem falle, wo zu dem genetiv eine apposition tritt, ist das alte gesetz durch die responsion durchbrochen: I, 5, 28 dauides sez thes kuninges; vgl. III, 10, 10 dauides sun thes gúaten.

Beim zusammentreffen eines substantivs nit zwei adjektiven gibt es folgende möglichkeiten: a) III, 6, 28 finf girstinu brot ist nach der alten regel betont, dagegen kommt in IV, 35, 21 thie zuéne richun thégana schon die accentresponsion zur geltung. b) Hier finden wir schon allenthalben gewichtsausgleichung: 1II, 22, 37 manigu uuérk guatu; II, 16, 38 mihil lón garauuaz; obenso in der correctur von P III, 6, 48 zuelif kórbi folle. c) In dem einen mir zu gehote stehenden beispiele finden wir accentresponsion V, 9,4 giséllm zuenc giate.

llier nuss ich schon bemerken, dass in den zahlreichen nominalgruppierungen, bei denen einer der drei bestandteile ein pron. possess., oder pron. demonstr. oder das wort selb ist, bereits die rhythmische accentresponsion oder die rhythmische gewichtsausgleichung zur herrschaft gelangt ist. Da derartige zusammenstellungen nun gerade bei Otfrid sehr häufig sind, so lassen sie die erwähnten rhythmischen gesetze als fast vollständig zur geltung gelangt erscheinen, während für die ältere wahl der stäbe nur verbältnismässig wenige beispiele vorliegen, die 
g-leichwol hinreichen, un den zusamnenhang von Otfrids accenten mit den stäben der allitterierenden dichtung vollstïndig zu erweisen.

Aucb in dem falle, wo ein substantiv mit einer apposition verbunden ist, von dor ein genetiv oder adjektiv abhängt, kommen schon die neueren gesetze zur geltung. So herrscht responsion in: I, 12,14 drúhtin krist guater; II, 2, 16 drühtin krist ther griato; ebenso in der corr. von P I, 7, 27 Johánnes druhlines druit, wo $\mathrm{V}$ gewichtsausgleichung hatte. Dagegen hat $P$ die alte betonung noch wider hergestellt in $I, 12,16$ sin múater mágad sconu.

Ein adjektiv mit abhängigem casus, zu dem ein adjektiv oder genetiv gehört, a) regelrechte betonung hat III, 18, 55 aller finfzug iaro; dagegen ist in II, 2, 37 follan gótes ensti rhythmische gewichtsausgleichung eingetreten. b) Rhythmische responsion findet sich in III, 7,6I thera kristes lera folle.

Von den unbestinmten adjektivischen quantitätsbezeichnungen manag, al und dem substantivischen filu gilt dasselbe wie in der allitt. dichtung: sie können voranstehen, olme den accent auf sich zu ziehon z. b. II, 16, 20 filu liebes; III, 9, 4 manag séltsani; I, 23, 14 elliu uuóroll; doch zichen sic es vor den accent zu behalten, wie das der regel entspricht.

Das verb hat weder vor noch nach dem substantiv einen accent, wenn das letztere keinen hat.

a) Vor dem substantiv: es sind der alten regel ontsprechend sowol betonungen wie III, 22, 46 ebonot. thin unfruati; I, 16, 9 deda si tho then githánc (vgl. V); III, 2(), 139 oba thu scounost thaz muat (vgl. V) u. s. w.; als II, 11, 42 rihla uns then sin hiar filu frám; III, 22, 22 thic éigun min io minna u. s. w. Beispiclo sind schr hijufig. Der erston betonungsweise entsprechend corrigiert auch P III, 15, 15 tho balun sine sibbon; dor zweiten entsprechend: II, 14, 81 tho quamun thie iningoron innan thes; II, 14, 13 so quaim ein unib thara tho. Doch auch hier ist das prinzip bisweilen durch herstellung der rhythmischen responsion durchbrochen: III, 6, 45 gibót tho druhtin sinen; I, 10, 27 Uuúahs thaz kind in édili; und dem entsprechend corrigiert $P$ IV, 7, 1 giang tho druhtin thanana. Für falsch halte ich die accentuation in P I, 25, 4 álfol spráh er uuorto. 
b) Nach dem substantiv ist die regel meist beobachtet, und es sind folgende möglichkeiten der betonung: I, 3, 21 thaz gumisgi al gizélle; 21), 11 thie bristi sie in ougtun; III, 8, 15 so ther liut tho zigiang u. $\mathbf{8}$. w. oder 1, 12, 26 in hirzen hugi thu inne; III, 16, 41 nir iu kind bisnidet u. oft. Sogar in cinem falle, wo ein starker rhetorischer ton auf dem verb liegt, wird keine ausnahme gemacht I, 1, 9 thaz then thio briah nirsmáhetin. Den entsprechend bat $\mathrm{P}$ mit recht nach der ersten betonungsiveise die schlechtere accentuierungsweise von $\mathrm{V}$ corrigiert: I, 4, 37 in ábuh irrentes; 14, 20 thaz kind ouh thárabrahtin; 20, 11 thaz fähs thánarouftin; III, 2, 3 Ein küning giciscot iz in uuar; 8, 13 thie undon blinenti; nach der zweiten betonungsweise: I, 3, 50 thie uuége riht er ino ubaráal; 3, 7 uuas er liut beranti ( $\mathrm{V}$ hat in diesem beispiele auch richtige betonung, aber nach der andern art); 17, 37 thia búrg nantun sie sár; 19, 8 thin múater tharafuaren; 22, 7 thia fira gientonun; II, 9, 17 thie män firnement álle; 9, 31 drühtin kos imo einan uuini; 12, 76 thia uuorolt pinoti; 14,52 finfi habotost thu iu; 14, 58 thaz thu hiar bita ouh suaches; 22, 42 thia fruma gibit er in sar; III, 6, 34 thio buah zellent uns thaz. Neben diesen zahlrcichen bcispielen der anwendung der älteren regel finclen sich aber auch solche, wo die rhythmische responsion des accents auf zweiter und vierter silbe das gesetz durchbricht, so in ersten halbverse I, 5, 27 got gibil imo uuiha, und demgemïss corrigiert $\mathrm{P}: 1,22,9$ thaz kind giduálla thia färt; II, 1, 13 er mano rihti thia náht; I, 16, 25 uuizzi thich imo ana sár; 17, 43 thia zit eisgola er fon in, und so fasse ich auch die correctur des stadiums $O_{1}$ auf in $I, 1,69$ zi nuzze grébit man ouh thár. Dieselbe responsion zeigt sich auch das gesetz durchbrechoud in zweiton halbverse I, 6, 3 thiu unirlun sia erlicho intfiang; cbenso fasse ich die correctur auf in I, 2, 23 thaz herza uuéist thu filu báz, und in 1, 4, 69 so got gisizta thia zit ist in $\mathbf{P}$ der letzte accent vergessen. Endlich durchbricht auch die rhythmisohe gewichtsausgleichung in cinigen fällen das grundgesetz: I, 26, 3 then brunnon reinota; 20, 9 zahari úzfluzzun; 11,32 zi gote uuuinsgenti; 5, 11 uuerk uuirkendo; 27, 65 spriu thánauuerre. Die accentuierung von PV in $\mathrm{I}, 17,34$ uuar krist gibóran uuurti ist falsch. - Zu beachten ist, dass das pronominale man von dem substantivum unterschieden 
werden muss; die betonung in I, 1, 1 in búachon man giméinti ist also ganz richtig (auch in I, 5, 13 ist man pronominal).

$\Lambda$ uch wenn das verb vor oder nach einem abbängigen infinitiv oder particip stebt, galt die regel, dass es den aecent nicht hat, wenn nicht der infinitiv oder das particip auch einen solchen trägt. Es entsprechen dem gesetzc also vor dem infinitiv u. s. w. wider zivei arten der accentuierung: I, 17,52 er uuólta nan irthuésben; 22, 59 er uuólta unsih léren; 5, 52 uuilit er gifáhan; 10,11. 11, 58. 23, 39 ni thàrf es man biginnan; 18, 11 uuir éigun iz firlazan; II, 21, 8 ni lízet uuergin uuánkon u. ö.; oder III, 18, 11 bigondun sie ántuurten; 20, 25 hiaz faran unásgan iz thar (vgl. II, 18, 23 far bisúani thih ir responsion in $I, 1,46)$. Welche von beiden accentuierungsweisen in jedem falle zu nehmen war, entschied Otfrid nach rhythmischen gründen. Nach der ersten art corrigiert $P$ in: I, 25, 8 unio mág ih biuuánen thanne mih; 26, 6 hiar mág ir lérnen ubaral; IV, 19, 64 móhtin giánabrechon; 7, 17 uuio se scóllun fáhan; II, 12, 35 scál siu irbéran meist; I, 28, 11 uuir unsih múazin sámanon; II, 7, 1 biginnu ih hiar nu redinon unio er bigónda brédigon; 22, 13 biginnet ánascouuon; 21,9 ni lázel fáran iu thaz mual; I, 11, 23 uuárun io ginánte; II, 6, 55 nu birun uuir gihuirsgle. In diesen fällen hat $\mathrm{V}$ die zweite (ebenfalls richtige) accentuierungsart; nach der zweiten corrigiert $\mathbf{P}$ nur in folgenden fällen; IV, 17, 15 ih mag giuuinnan heriscaf (V mág giuuinnan); I, 5, 39 haben ih giméinil (V háben); V, 23, 54 thiz scal sin io thes githig (V scál sin); I, 24, 13 uuir sculun thiu uuort ahton (V scullun); 23, 39 so er sih biginnit bílgan (V biginnit); IV, 35, 24 sie uuarun uuártenti (V uuárun). Die responsion der ersten zur dritten silbe bewirkt eine abweichung von der regel: I, 17, 67 sie móhtun bringan mira; I, 10,9 ih scil thir sagen kind min; 14, 22 ih scál iu sagen uuunlar (vgl. P I, 12, 7); und ebenso corrigiert P in: I, 4, 55 uuio mág ih unizan thánne; 18, 4 ih mág iz lobon hárto; auch dio rosponsion der zweiten zur vierten silbe $I, 11,7$ in thin se uuóllen haben lib. Dagegen ist die accentuierung von $\mathrm{V}$ richtiger als die von $\mathrm{P}$ in: II, 14, 29. III, 2, 4 uuaz mag ih zéllen thir ouh mir; IV, 7, 10 so frám sie iz mugun bringan; III, 4, 38 er hiaz mǐ gángan mit thiu; IV, 37, 3 ioh lazan sin thaz sláfan; V, 2, 30 bigán er sulih rédinon. 
Nach dem infinitiv sind folgende zwei arten der accentuierung richtig: I, I, 10 then lésan iz gilusti u. ö. oder IV, 6, 44 ioh uuérdan ni mohti und oft. Nach der ersten accentuationsweise corrigiert $P$ nur in $111,14,73$ theih al irzéllen ni mig; nach der zweiten aber: I, 11, 82 thuz thu irrimen ni maht; 11,55 druihtin quiman uuolla (V queman uuólta); II, 4, 66 then uneg ther fáran uuolle; 4, 89 thic inan béton uuollent; III, 15,23 ther sih offonon scal; IV, 11, 10 zi imo fáran scolla (V faran scólta); 11, 33 ob iz súlih uuesan scal (V scál); I, 17,5 thes mera ih ságen nu ni tharf; 2, 32 irfirrit uuerde bálo sin; 1, 7, 2 sámanon bigonda; III, 20, 167 firméinsamot uuari. Der responsion wegen ist das gesetz durchbrochen in I, 8, 4 theiz álles uuesan móhti.

Das adverb 1) braucht weder vor noch nach den nomen, wenn dieses accentuiert ist, den accent auf sich zu ziehen; ist das nomen nicht accentuiert, so kann auch das vorhergehende oder folgende adverb den accent für gewöhnlich nicht auf sich ziohen. Statthaft sind also folgende verbindungen, die ich mit je einem beispiele aus Otfrid belege: a) I, 1, 20 theist góma filu réini; b) 1, 27 ioh hárto filu kléino; c) IV, 5, 8 ist húarilinaz harlo; d) I, 1, 107 flizig filu hárto; e) II, 7, 27 thia fruma uns füntan filu fram; f) H 11 firdanan unéiz ih filu mih. Nach a) ist in $\mathrm{P}$ corrigiert IV, 2, 8 filu scin (ferner I, 1, 53); nach b) in I, 1, 59 so sáma chuani (vgl. I, I, 62); nach c) in I, 12, 25 frima frua; nach d) in I, 13, 8 filu frúa. Man sicht, dass die betonung des adverbs nach sehr freien gesetzen erfolgt, ganz ontsprechend soinem sehr verschiedenen bedeutungswerte im satze. Merkwlirdig sind noch eine anzahl von beispiclen, in denen das adverb, besonders filu, vor dom nicht accentuierten nomen don ton. trïgt. Ich fuhre zunächst an I, 1, 15 filu slehtaz; 1,63 fïlu chuani; 1, 67 filu feizzit; 1, 75 filu redie; 1,90 filu herten; 20, 28 filu hohen (vgl. auch I, 1, 62 so sáma balde mit 1,59). In diesen fällen haben wir wol mit Rieger (s. 27) den aufang einer compositionsbildung zu erkennen. Ausserdem findet sich dasselbe

1) Ich glaube hier der regel Riegers (s. 26) eine richtigere fassung auch ftir dic allitterieronde poesie gegeben zu haben; natïrlich muss da für 'accentuiert' gesetzt werden 'den stabreim tragend'. 
in einigen fällen bei harto, wo diese betonung ganz offenbar durch den rhetorischen accent bedingt ist und eine besonders emphatische herrorhebung stattfindet; vgl. die correcturen von $P$ in: I, 5, 42 hórto filu uuahaz; 18, 25 hárto bistu herti; 2, 24 hárto kundera; 4, 1 hárto firdanes; III, 20, 104 hárto ungimah. Ebonso ist die correctur von $\mathrm{P}$ in III, 5, 14 uuérgin missilichan aufzufassen.

Ueber die präpositionaladverbien beim verbum sind die beispiele schon Einl. no. 194-196 gegeben, auf die ich verweise; doch wird in einigen einzelbeiten die dort gegebene erklärung der accentuierungen nach den hier aufgestellten grundsätzen $\mathrm{zu}$ modifizieren sein.

Die pronominaladverbien vor und nach dem verb tragen nur den accent, wenn ein rhetorischer ton auf sie fällt. Demgemäss corrigiert $P$ richtig, z. b.: I, 9, 9 stuant tho thar; 16, 8 kuimta thar; 16, 17 kuindtha thar; 19, 22 ladon thanana; 1, 5 tharana dátun; 11, 27 uuarun thanana (vgl. 12, 15); II, 11, 58 hiur gispráh; I, 26, 7 lisist hiar. Feblerhaft ist die betonung von VP in I, 15, 2 thiononti thár. Ueber das thár in $I, 17,60$ $P$ vgl. Einl. s. 166. Durch emphase der frage erklărt das vuánana in $\mathrm{I}, 1,33$.

Sonstige adverbien des orts ziehen vor dem verb gern den accent an sich; während sie nach den verb ihn leicht einbussen. Lehrreich sind die correcturen von $P$ in: I, 1, 52 bifóra lazu; 27, 70 héimortes fúarin aber III, 10, 12 ther diufal ist iru inne. - Fur adverbien der zeit sind die correcturen in: I, 16, 9 deda si tho; I, 2, 9 thiu er deta thó; 13,6. 8 ougta uns zi érist; 16, 4 gihialt si frám beachtenswert, besonders auch III, 1, $7 \mathrm{Ni}$ scribu ih nu in alauuár, wo der dichter den gegenwärtigen abschnitt den fruheren ontgegenstellt, in lenen or dem evangelischen texte folgte.

Bei sonstigen adverbien vor und nach dem verb uibt der ihnen anhaftende rhetorische ton einen grossen einfluss. So ist die correctur von $P$ in III, 12,6 giuuisso sagel mir iz ál leicht begreiflich. Ferner verglciche man die correcturen von $P$ in: I, 1, 14 so thih es uuola lustit; 26, 12 ther douf io uquola thihe mit I, 1, 123 so uuer so uuola uuolle und den correcturen ron $P$ in: I, 1, 43 uubla drahton (= ganz richtig); 14, 7 ist uuíla (= ganz gewiss) so giméinit; 27, 31 sos er uuóla konda 
(= so gut nur immer er es vermochte); ferner die correctur von $P$ in: I, 1,67 harto ist iz giuuéizzit; 13, 15 harto sie iz intrielun; 17, 78 harto ilente; II, 6, 5 hurto ságeta mit I, 4, 65 so hárto bist firmónanti; 23, 52 hárto gislimit; ferner die correcturen von $P$ in: $1,1,37$ theiz scono thoh gilute (auch in v. 39 ist scóno zu schreiben); II, 22, 12 scóno giuuérien; I, 15, 3 réhto er lebeta. So ist auch $111,8,25$ zu schreiben lúlo irháretun. - Für das dem verbum nachfolgende adverb vergleiche man die correctur von $\mathrm{P}$ in $\mathrm{I}, 18,4$ lobon hárto (wo die rhythmische responsion von einfluss war) mit denen in I, 19, 11 hügi filu harlo; 21, 12 hinlarquam er harlo; 27, 44 sie insázun iz harlo; ferner dic correcturen: I, 27, 22 frágetun sie auur; 27, 35 spráchun sie unur mit 27, 43 bi thiu frágetun se áuur mera (zu mera vgl. dic corr. in II, 12, 29); ferner 17, 32 niazen gerno; III, 8, 32 griazla baldo mit II, 4, 3 fástela úrnolo und III, 16, 45 nirdéilet rinrehto (in beiden fällen konnte der accent auf dem adverb nicht umgangen werden); vgl. danach I, 17, 32 gihorlun úngerno.

Von zwei in beliebiger weise verbundenen begriffswörtern kann das erste ohne das zweite, nicht aber das \%weite ohne das erste den accent tragen. Beispiele sind sehr zahlreich bei Otfrid. Ich gebe also für jede der vorhandenen möglichkeiten nur cines: a) I, 22, 62 in uuáhsmen ioh giuuizze $(6,2.7,17$. 8, 4.10. 10, 17. 11, 7. 9. 14. 21.46. 52. 16, 13.19. IV, 13, 23. 18, 1. 20, 27 u. oft); b) I, 14, 16 thiu thiarna noh ther ira sun. Nach der ersten betonungsweise, welche die gewöhnlichere ist, corrigiert P: I, 15, 42 úbil odo uuir; II, 6, 22 gúat ioh úbil; II, 16, 13 thúrst ioh húngar; $\mathrm{V}, 19,48$ kind noh quena; 20, 16 ir noh sidor; IV, 22, 16 liob ioh súazi; vgl. auch V, 17, 9 giuuált ioh gótes krefti und bei mohr als zwoi gleichartigen nominibus IV, 5, 2 in férti int in gánge ioh in thero liulo sange; ebenso IV $, 4,15.16$, wo in $P$ nur ein fluchtigkeitsfehler ist. Nach der zweiten, seltncren art corrigiert P: I, 11, 16 stérron odo manon; 21, 16 mil góte ioh mil manne; II, 22, 23 minnoron noh mera; III, 6, 10 uuib inti gomman; IV, 23, 38 in lib ioh dod. Der responsion wegen findet in $\mathrm{VP}$ eine abweichung statt in: IV, 15, 4 in got gilóubel ioh in mih; IV, 23, 38 in lib ioh dod hiutu. Dic accentuierung von $\mathrm{PV}$ in $\mathrm{IV}, 20,34$ ist iu kind nales mir und die von $P$ in $11,13,2+$ thaz sihit er ál ioh hórit ist 
falsch. - Auch gegenuberstellungen wic IV, 33, 9 fon séx/u unz in nóna gehören hierher, und darnach corrigiert $P$ in $\mathrm{V}, 12,75$ ni uuédar ana ánder.

Ueber die fähigkeit der verecliedenen gattungen der pronomina, der präpositionen und conjunktionen, den accent zu tragen, habe ich schon in der Einl. zur ausgabe gesprochen, zu dem durfte es bei diesen mit grö̈sserer schwierigkeit verkniipft sein, den zusanmenhang der accente mit den stïben der allitterierenden poesie klar nachzuweisen.

Welches ist nun das ergebnis der bisherigen untersuchung? Mit sicherheit lässt sich erkennen, dass Otfrid die bestimmungen, welche für die wahl der stabenden wörter galten, auch bei seiner accentuierung zu grunde legte. In der grossen masse des vorhandenen materials lässt sich das deutlich bemerken. Allein dieses grundgesetz findet sich schon häufig durebbrochen um rhythmischer rücksichten willen, wie sie durch die lateinischen hymnen dem dichter nahe gelegt wurden. Hierin sehen wir das neue dichterische prinzip allmählich das frtibere verdrängen. Wir sprachen von einer rhythmischen responsion und einer rhythmischen gewichtsausgleichung. Jene findet in den halbversen mit zwei accenten statt, also in den ersten der verse; diese in den halbversen wit einem acconte, also besonders in den zweiten. Jene ist doppelter art: entweder ontspricht der accent auf der ersten hebungssilbe einem solchen auf der dritten, oder einer auf der zweiten einem accente auf der vierten hebungssilbe. Wie diese beiden rhythmischen gesetze allmühlich in den correcturen von $P$ inmer melsr durchdringen, habe ich schon Otfrid Einl. s. $149 \mathrm{ffi}$ gezeigt. Die rhythmische gewichtsausgleichung findet hauptsächlich dann statt, wenn das letzte wort des halbverses drei hebungen triagt, z. b. manno éristo, manno liobosto, ferner auch wenn die beiden letzten wörter, die fast wie ein compositum zusammengehören, drei hebungen tragen (\%. b. II, 12, 24 thie fol sin gúules uuillen; 14, 6 thes kindes háft uuurti).

Es drängt sich nun die frage auf, ob die vertcilung der accente bei Otfrid nach eben den grundsätzen stattgehabt hat, wie die der reimstïbe in der allitterierenden dichtung.

Ich gehe von den Einl. 8. 76 aufgestellten zwei fundamentalsaitzen von Otfrids accentuierung aus. Den zweiteu der- 
selben, Otfrid habe nie mehr als zwei accente in den halbvers zu setzen beabsichtigt ${ }^{1}$ ), werde ich aus seiner negativen form in positive bestimmungen umzusetzen versuchen.

Fir die allitterierende dichtung (s. Litteraturgesch. und Gramm. s. 454) gilt das gesetz, dass im zweiten halbverse nur ein stabwort, der hauptstab, sein darf; nur in einigen seltnen fällen scheint es, dass zwei stabwörter im zweiten mit zweien (nie mit einem) im ersten allitterieren düren. Schon beim flüchtigen übersehen einer anzahl von Otfrids versen fällt uns auf, dass im zweiten halbverse sich vorzugsweise meist ein accent findet. Dass dies nicht zufall ist, zeigen folgende tatsachen. $P$ hat in einer grossen anzahl von fällen, nämlich in 858 zwciten halbversen, einen accent, wo $\mathrm{V}$ noch deren zwei hat $\left.{ }^{2}\right)$. Dabei sind nicht eingerechnet die unten zu erwähnenden zablreichen fälle, wo $P$ nur cinen accent im zweiteu halbverse setıt (währeud $V$ noch zwei hat), weil im ersten halbverse auch nur ein accent steht. Nun kommen allerdings auch eine anzabl von fälleu (382) vor, in denen $P$ die, an sich nach obigen prinzipien ja immerhin statthaften, zwei accente für den zweiten halbvers wählt, wo $\mathrm{V}$ deren nur einen hat. Fehlerhaft ist in allen diesen 382 fällen der zwiefache accent an und fur sich nicht, da auch im ersten halbverse jedes mal zwei accente stehen, in 52 dieser fälle hat sogar zugleich im ersten halbverse die vermehrung der accente auf $\mathrm{zwei}$ statt gefunden, während $\mathrm{V}$ nur einen hatte, in diesen 52 fällen war also dic vermehrung der accente im zweiten halbverse anlass zu der vermehrung im ersteu, da zwei accente im zweiten halbverse nicht mit einem im ersten correspondieren durfen; gleichwol werden von der zahl 382 noch crhebliche abzlige zu machen sein. Es gibt darunter fälle, wo der eine accent gar kein

1) Wenn $P$ noch 278, $V$ noch 354 (von denen $55 \mathrm{P}$ und $\mathrm{V}$ gemeinschaftlich) halbverse mit 3 und 4 accenten hat, so sind das correcturen; die ungiltigen accente sind meist leicht nach dem oben gesagten herauszuerkennen.

2) Um aus den nun folgenden zahlenangaben eine anschanung des prozentsatzes entnehmen zu können, beachte man, dass Otfrids gedicht aus $L+S=144$, buch $I=1240$, buch $I I=1244$, buch $I I I=1576$, buch IV $=1572$, buch $\mathrm{V}=1472, \mathrm{H}=16 \mathrm{~S}$, in summa $=7416$ versen besteht. 
versaccent ist, sondern den auftakt markiert (Einl. s. 166) z. b. I, 9, 31 ther thaz uuas máchonti. In andern ziemlich zablreichen fällen liegt die sache so, dass eine besserung après la lettre stattgefunden hat, ohne dass der ältere accent getilgt wurde, z. b. I, 14, 20 thaz kind ouh thárabrahtun, wo durch dic Thesserung eine rhythmische gewichtsausgleichung hergestellt werden sollte. Manche fälle beruhen auch auf entschiedmem irrtum, so I, 10,12. In anderen fällen stellen die zwei accente von $P$ allerdings eine entschiedene besserung dar, so II, 2, 31. 14, 81. III, 8,13 , wo die ursprünglichen stabgesetze, oder IV, 31, 17. V, 23, 80. 106.116. 146, wo die rhythmische responsion von einfluss waren. Immerhin aber ergibt sich, dass die fälle, in denen $\mathrm{P}$ im zweiten halbverse éinen accent (gegen 2 in $V$ ) setzt, mindestens um das vierfache überwiegen. Nun könnte man allerdings hervorheben, dass unter jenen 852 fällen auch 105 sind, in denen in $\mathrm{P}$ die herabsetzung von zwei accenten auf einen (gegen V, das zwei behält) zugleich in ersten halbverse stattfindet, allein das ändert an der tatsache ja nichts, dass in der zeit der reiferen arbeit Otfrid entschieden nur einen accent im zweiten halbverse bevorzugte. In vielen fällen wurde sogar sicher die besserung im zweiten halbvers dadurch hervorgerufen, dass im ersten ein accent gestrichen wurde, z. b. I, 24, 2 unio se ingíangin alle themo égislichen falle; II, 4, 104 u. o. - Im ersten halbvers (ich zäble auch hier uberall die fälle noch mit, die unten bei der responsion zwischen zweitem und erstem halbvers zur sprache kommen) hat $P$ in 104 fällen zwei accente, wo $V$ nur einen hat, und zwar meistens, um den im zweiten halbverse stehenden zwei accenten ihr cntsprechendes gegengewicht zu geben, oder auch, um durch den ersten auftakt, resp. die schwebende betonung anzudeuten, wie I, 18, 33 fárames so thie ginoza ${ }^{1}$ ). Dagegen bat in 118 fällen, $\mathrm{P}$ einen accent im ersten halbverse, wo V deren zwei hat. In all diesen fällen entspricht denselben éin accent im zweiten halbverse; die fälle, wo in $P$ gegen $\mathrm{V}$ ein accent im ersten zweien im zweiten halbverse

1) Wo bei Otfrid drei accente stehn, ist bisweilen auch beabsichtigt, die scansion des verses deutlicher hervortreten zu lassen, so III, 6, 31 . 18,3 u. $\ddot{0}$.

Beiträge zur geschichte der deutschen sprache. VIII. 
gegenübersteht, sind nicht mitgerechnet. Diese 118 fälle sind besonders lehrreich, denn da kein metrischer zwang vorlag, den zweiten accent zu setzen, so ist der grund in der betonungsfähigkeit der wörter zu suchen; nur in ganz vereinzelten fällen liegt ein vergessen in $P$ vor. Es fällt auf, dass wenn auf ein adjektiv ein substantiv von nur zwei hebungen folgt, gern nur das erstere den ton bekommt (vgl. die correcturen in IV, 23,32. V, 1,6. V, 23, 74) ebenso bei vorangehendem genetiv $(\mathrm{V}, 23,100)$.

Dass also zwei accente im zweiten halbverse noch statthaft sind, haben wir gesehen, wenngleich Otfrid auch in den späteren stadien der textentwicklung die accente im zweiten halbverse möglichst auf je einen zu beschränken sucht. Allein man sollte, den gesetzen der stabreimenden dichtung folgend, erwarten, dass niemals zwei accente im zweiten halbverse mit einem im ersten correspondierten. 'Tatsächlich tritt auch in $\mathbf{P}$ das streben hervor, wo in $\mathrm{V}$ jene fehlerbafte zusammenstellung sich findet, dieselbe zu heben. Es geschieht das gewöhnlich, indem im ersten halbverse ein accent hinzugesetzt wird (in 227 fällen), aber auch indem in zweiten ein accent weggelassen wird (in 154 fällen), oder endlich indem im ersten halbverse ein accent hinzu, in zweiten dagegen einer weggelassen wird (in 60 fällen). Bisweilen geschieht es auch so, dass statt des einen accentes im ersten halbverse von $P$ zwei ganz andere gesetzt werden (ich habe mir 6 fälle aufgeschrieben). Im ganzen hat also $P$ in 447 fällen gegen $V$ die richtige accentuierung hergestellt. Das gesetz war so strenge, dass man selbst minderbetonte wörter accentuierte, um es aufrecht zu halten (vgl. I, 20, 14. II, 4, 30. 7, 21. 12, 26. III, 6, 47. 20, 9 23,9 . IV , 29, 18. V, 3, 5 u. ö.), oder dass man einem worte den accent entzog, wo er des rhythmus wegen ungern entbehrt wird (I, 33, 1. III, 25, 32. V, 23, 118 u. o.). In I, 1, 53 ist z. b. die correctur offenbar im zweiten halbverse deshalb gemacht, weil sie im ersten erfolgt war. Nun bleiben noch die (320) fälle zu besprechen in denen sowol bei $V$ als bei $P$, und die 252 anderen, in denen sogar bei $\mathrm{P}$ gegen $\mathrm{V}$ zwei accente im zweiten mit einem im ersten halbverse correspondieren. Unter den zuerst erwähnten 320 fällen finden sich allein 129 solche, in deneu der erste halbvers auf ein wort mit drei vershebungen 
ausgeht, das also naturgemäss ein anderes hochbetontes wort nicht neben sich duldet ${ }^{1}$ ), in etwa 50 anderen beispielen soll ein wort des ersten halbverses besonders vor allen andern hervorgehoben werden, in 17 andern fällen steht überhaupt nur éin wort im ersten halbverse, das den accent tragen kann, endlich in 16 jener 320 verse würden, wenn noch ein zweites wort den accent erhielte, zwei accentuierte silben aufeinanderfolgen, was Otfrid sichtlich vermeidet. In einigen fällen kann man aber auch mit sicherheit annehmen, dass, nach dem sonstigen gebrauch Otfrids zu urteilen, im ersten halbverse ein accent hinzugesetzt, oder im zweiten nach erfolgter correctur der ungültige nicht deliert worden ist. In einer anzahl von fällen dürfte auch der vermisste zweite accent des ersten halbverses in den diakritischen accenten von $i u$, $i \dot{o}$, iúer u. s. w. stecken. Nach abzug aller dieser fälle bleiben noch nach meiner zïhlung 77 beispiele in denen keine solche entschuldigung vorliegt; häufig, etwa in der hälfte dieser zahl, tritt die absicht hervor, wenn substantiv und adjektiv oder substantiv mit genetiv neben einander vorkommen, nur das zuerststehende dieser beiden $z u$ accentuieren (s. oben). Jedenfalls lassen alle diese beispiele erkennen, dass Otfrid aus rhythmischen, sprachlichen, rbetorischen grinden nicht umbin konnte, das gesetz, welches er der stabreimenden dichtung entlehnt hatte, bisweilen schlafen zu lassen; allein es mag dies bei der verhältnismässig verschwindend kleinen zahl der fälle der punkt gewesen sein, an dem er, wenn es ihm verstattet gewesen wäre, gern noch seine nachbessernde hand hätte wirken lassen.

Wichtig für diese auffassung wird die betrachtung der 252 fälle sein, wo $P$ (gegen V) noch zwei accente im zweiten éinem im ersten halbverse gegenuberstellt. Diese stellen sich äusserlich ganz anders dar, als jene eben besprochnen, die VP gemeinschaftlich haben. In einer grossen anzahl von fällen erklären sich diese abnormitäten so, dass, nachdem sich im zweiten halbverse das bedürfnis eines zweiten accents heraus-

1) Hierher könnte man vielleicht auch einige fälle zählen, wo substantiv und adjektiv fast zu einem compositum verschmelzen, wie I, 24, 6 . IV, 30. 33. V, 8, 13. 22, 5. 23, 43 . 
gestellt hatte, Otfrid im ersten die gleiche vermehrung der accente nicht vornehmen konnte, entweder - was am häufigsten war -, weil das letzte wort des ersten halbverses drei hebungen trug (vgl. I, 4, 40. 5, 40. 12, 20. 17, 32 u. s.w. u. s. w.; compositionsähnliche zusammenstellungen in demselben falle I, 15, 8. 36. 26, 12 u. s. w.), in welchem falle, wie wir oben geseben, der halbvers in der regel nur einen accent tragen durfte; oder weil kein zweites, accentfähiges wort vorhanden war, oder auch weil mebrere solche da waren, zwischen denen Otfrid noch schwankte, oder endlich weil im ersten halbverse ein wort einen besonders nachdrücklichen ton tragen sollte. Bisweilen liegt auch augenscheinlich der fall vor, dass Otfrid den einen accent im ersten halbverse einfach zu setzen vergass, oder dass, nachdem bei der tibercorrectur eine änderung der accentstellung im zweiten halbverse vorgenommen war, der ältere accent nicht deliert wurde. Jedenfalls bleiben nur äusserst wenig fälle übrig, wo die correctur nach den oben dargelegten grundsätzen nicht mit leichtigkeit vollzogen werden könnte. Eine solche verbesserung darf natürlich nicht vorgenommen werden an den stellen, wo, wie wir eben bei besprechung der 320 fälle zeigten, die entsprechung von einem accente im ersten halbverse gegen zwei im zweiten stathaft, resp. fur Otfrid unvermeidlich war.

Wir steben hier wider an einem punkte, wo deutlich zu sehen ist, dass das werk in seiner jetzigen gestalt fur den dichter noch unfertig war, und wo von demselben jedenfalls noch besserungen vorgenommen worden wären, weun ihn, wie ich vermutet habe, der tod nicht seiner arbeit entrissen hätte.

\section{Zu Otfrids leben.}

Es existieren zwei umfangreiche listen Weissenburger mönche in dem noch nicht herausgegebenen verbrtiderungsbuche von St. Gallen und in dem von Reichenau '). Die St. Galler liste steht auf p. XL-XLII des codex und enthält in neun columnen (die ich nach meiner ausgabe mit den nummeru

1) Eine ausgabe von mir steht nächstens in den monumenta Germaniae bevor. 
210-218 bezeichne), mit je 32 namen die Weissenburger mönche. An der spitze von col. 210 stehen Justolfus eps. Perinharius eps. Folcuuicus eps. Ato eps. Otger eps. Grimaldus abb. Benedictus eps. Der letztere (das St. Galler stluck ist (iie abschrift einer älteren originalliste) ist in der urschrift wahrscheinlich nachgetragen gewesen, in der abschrift aber als gleichberechtigt aufgenommen worden, obgleich or gar kein Weissenburger abt war, wic die zwei series abbatum Wizanburgensium zeigen in Scriptores XIII s. 320.1) Wir werden demuach die erste abfassung dieser liste in die erste periode der abtschaft Grimalds zu setzen haben (833-840). Der name Otfrids begegnet nun in sp. 215 und 217, doch ist letzteres sicher eine widerholung, da auch sonst namen, der früheren columnen in sp. 217 sich widerholt finden. Ich vermute, dass der inhalt der sp. 217 und 218 in dem ursprünglichen exemplar des verzeichnisses spätere nachtragung, etwa von defunctis, war, die dann in der abschrift nicht mebr gesondert gehalten wurde. ${ }^{2}$ ) Noch lehrreicher ist die liste, welche sich auf $p$. XLVI und XLVII des Reichenauer verbriderungsbuchs findet. Die acht spalten dieser zwei seiten bezeichne ich nach meiner ausgabe mit col. 177-184. Es finden dort folgende entsprechungen mit dem St. Galler verbrtiderungsbuche statt. Auf sp. 184 finden wir die namen von col. 214, 24 bis 217, 23 wider. Die namen 218,1-12 sind, als aut 184 kein platz mehr war, am schlusse von sp. 183 nachgetragen. Da nun an der spitze der ältesten namen von sp. 183 Folcuingus eps. ct abba steht, so mitissen dic namen von sp. 184 (unter denen auch Otfrid steht) und die letzten von sp. 183 jüger sein als

1) Der band ist noch nicht erschionen, doch wurde mir durch herrn gcheimrat Waitz' giite einblick in dio aushängelogon verstattet.

2) Ich fiuge zu der in der einleitung meiner ausgabe gegebnen aufsiihlung der stellen, wo der name Otfrid erwähnt wird, noch die folgeuten: Im St. Galler verbrỉderungsbuch 1,34 und 243,1 als münch von śchönenwert, 157, 38 und 343,24 als benefactor, im Pfäverser verbrülerungsbuch 115, 6 als mönch von Schienen, im Reichenauer als presb. 16,5, als defunctus von Reichenau 25, 17, als Fuldaer mönch 134, 24, als Kemptener 160, 12, Weissenburger 1\$2, 30. 184, 22. 184, 32. 252, 19. 253, 22, von Schienen 334, 10, vom cocnob. Rasbaacis 272, 14, aus dem mon. s. Petripuellaris 375,24 , als benefactor 353,9 . 391, 27. 393, 20. 463, 15. 511, 5. 26. 5it, 26. 605, 6. 606, 6 . 
dieser (der nach Zeuss trad. Wizanb. p. XIV. XV in urkunden von 828-830 begegnet, und nach dem Reichenauer totenbuche XVII. Kal. Apr. starb); ich vermute dass sie aus der ersten periode der abtschaft Grimalds (833-840) stammen. Diese vermutung wird durch einen andern umstand zur gewissheit erhoben. Es findet sich nämlich auf der 3. und 4. spalte der s. LXIV (ausg. 252. 253) des Reichenauer verbrtiderungsbuchs (welche auf sp. 1 und 2 Nomina fratrum de coenobio quod Senonicas nominatur enthält) noch ein nachträgliches register der Weissenburger mönche, und zwar auch die namen aus sp. 214-217, unter ihnen auch Otfrid und an ihrer spitze Grimaldus. Es ist also nicht zu zweifeln, dass Otfrid schon in der ersten periode von Grimalds abtschaft Weissenburger mönch gewesen ist. Doch unsere verbrüderungsblicher geben uns noch weitere auskunft. An der spitzc von sp. 177 stehn iustulfus eps. gerboh abb. bernharius eps. ct abb., dann folgen in sp. 177 und 178 namen aus col. $210-212$, in sp. 179 nameu aus col. 212 u. 213, in sp. 180 verschiedene namen aus $210-214$, in sp. 181 einzelne namen von $210-214$, besonders aber von 215 , in sp. 182 zerstreute namen aus 211-217. Sicher aber finden sich in sp. 181 und 182 namen aus sp. 184 und 183 widerholt, und \%war offenbar in derselben rcihenfolge, so dass wir an deren identität nicht zweifeln können, und zwar steht gegen ende von sp. 182 auch Otfrid. Es wird also die annahme gerechtfeitigt scin, dass wir, besonders in sp. 182, mönche aus der abtschaft Gerhohs (in Urkunden von 819-826 erwähnt) und des Folewig (in Urkk. von $828-830)$ vor uns haben, unter denen sich viele befinden, die auch Grimalds regierung noch erlebten. Betrachten wir nun die an die spitze der col. 177 gestellten abtsuamen, so fällt deren reihenfolge auf, die ganz gegen die sonst in den confraternitilten Ubliche gruppierung verstösst (nach welcher dor jungste der äbte, unter dem die verbrtuderten mönche eingetragen wurden, an die spitze gestellt, indem ibm dic anderen älteren äbte des klosters in absteigender reihenfolge sich anschliessen). Es ist also wahrscheinlich, dass Gerhoh in der originalliste nachgetragen ist (auch das Reichenauer verbrtiderungsbuch ist eine abschrift älterer listen, und zwar ganz, während in den ersten fascikeln des St. Galler noch cinige der ursprilinglichen aufzeichnungen erhalten sind). Ich glaube also, dass in sp. 177-180 
und anfang von 181 die mönche unter Bernhari (in urkk. von $811-825$; vgl. auch simson, Ludwig d. Fr. I, 236 f.) aufgezählt, in der zweiten hälfte von 181 aber und ganz 182 die von Gerhoh. Dic abtliste des St. Galler verbrliderungsbuchs bietet indess auch noch einige schwicrigkciten. Es fehlt darin Gerhoh, während ein Ato eps. darin steht, der nach ss. XIII, 320 vie in Weissenbur abt gewesen ist. Sicher haben wir in dem letzteren eine nachtragung der originalliste zu sehen, dio in der erhaltenen copie unbedenklich in der reihenfolge der Weissenburger äbte aufgenommen wurde; aber warum der erstere weggclassen ist, vermag ich nicht zu erkennen. Das ergebnis fiir unsern zweck ist: Otfrid war schon unter Folkwig, vielleicht schon unter Gerhoh, mönch in Weissenburg, und als solcher auch noch im ersten teile der abtschaft Grimalds dort anwesend. Es tritt also die nachricht des anonymus in der series abb. Wizanb. ss. XIII, 320, nach der Otfrid unter Volcoltus eps. et abba (vgl. Einl. s. 18) lebte, der als vorgänger des Gerhoh genannt wird aber sonst nicht bekannt ist, in neues licht. Nun finden sich in der Weissenburger urkunde vom 16. juli 837, der schenkung des Heinrich und Albrich (Zeuss s. 154 no. 166) als zeugen eine anzahl namen, die sich teilweise auch auf sp. 215 des St. Galler verbruderungsbuchs finden, wir werden in diescm umstande also eine neue bestätigung unsrer chronologischen bestimmung jener liste finden. Die urkunde vom j. 840 (Zeuss s. 140 no. 151), die schon unter Otger ausgestellt ist, durfen wir, obgleich auch hier oinige zeugennamen mit der sp. 215 ubereinstimmen, nicht herbeiziehen, da mit dem in folge einer grossen politischen veränderung geschehenen abtwechsel offenbar auch eingreifende veränderungen im klosterpersonal ror sich giengen.

Nun noch einige rereinzelte kleinigkeiten zu Otfrids leben. Den gedanken, der in Fuldaer urkunden erwïhnte Otfrid sei vielleicht der dichter, halte ich nicht mehr aufrecht, da in den nunmehr vollständig ${ }^{1)}$ herausgegebnen annales necrologici Fuldenses (Script. XII, 175) cin Otfrid presb. als im jahre 842 in Fulda gestorben erwähnt wird.

1) Die friihere ausgabe bei Böhmer fontes III s. $155 \mathrm{ff}$. enthält nur eine dürftige auswabl. 
Dass man von einen wolwollenden und freundschaftlichen auftreten des bischofs Salomo I. gegen St. Gallen etwas wisse, hätte Meyer von Knonau (Forschungen zur deutschen Geschichte XIX s. 188) nicht leugnen sollen. Man denke an dic an verfolgung grenzenden bedrtickungen, welche die abtei seit der mitte des achten jahrhunderts von den Constanzer bischöfen erfahren hatte, die sich den besitz derselben anmassten. Der Constanzer bischof, welcher in diesem punkte auf die rechte seines stuhles verzichtete, muss natuirlich wolwollen gegen die abtei gehegt haben, und wir könnten dies annehmen, selbst wenn wir nicht den bericht über den förmlichen abschluss der streitigkeiten bei Ratpert besässen, der den entschiednen cindruck wolwollenden entgegenkommens auf Constanzer seite macht. Auch sonst hat aber Salono dem kloster woltaten erwiesen, indem er die heiligerklärung Otmars beförderte, und wenn auch diese tatsache nicht als beweisend gelten soll, so hätte doch Meyer von Knonau wenigstens dem bericht der von ihm selbst edierten vita $\mathrm{S}$. Otmari von Iso rechnung tragen muissen, welcher den Salomo in seinem auftreten bei den schwebenden verhandlungen p. 48 (M. v. Kn. s. 117) als benivolus bezeichnet und von ihm ferner s. 49 (121) sagt: praesul benignus orationibus fratrum ac benedictione munitus monasterium laetus egreditur, nachdem nämlich die feier zu ehren Otnars stattgehabt hat. Derselbe gelehrte verwirft meine vermutung, Salomo sei zwischen 820 und $\$ 39$ klosterlehrer in St. Gallen gewesen, als unsicher, statt, wie billig, sie auf ihre wahrscheinlichkeit zu prüfen. Ls hebt das schweigen Ratperts hervor, allein dies argumentum ex silentio ist jedenfalls misslich. Dic positiven nachrichten aber einzelne personen jener zeit sind nicht zahlreich. Nur durch combination und versehiedenartige beleuchtung kann man einige klarheit in diese zeiten bringen, gleichwie man verblichene handschriften immer wider und wider bei andrem lichte betrachtet und die strablen bald von dieser, bald von jener seite darauf fallen lïsst, um endlich nach verschiedenartigen vermutungen eine zu treffen, welche einem wahrscheinlich dunkt und der gesammtheit der von der stelle erhaltenen eindrücke am meisten entspricht. Wie man da nicht sagen kann, es steht da geschrieben, wol aber vermuten, es habe einst da gestanden, so 
kann ich auch hier nicht sagen, da und da stcht Salomo als magister scholao aufgezeichnet, wol aber, es ist wahrscheinlich, dass er oin solcher gewesen ist. Den zweiten aufenthalt Otfrids in St. Gallen habe ich nicht aus der anmerkung des lldefons von Arx erschlossen (obwol diese bei ihren detaillierten angaben - el fluhrt an codd. 561 p. 113 , no. 446 p. 50 et 79 p. 728 - nicht einfach iibergangen werden darf, zumal in der antwort auf meine schriftliche anfrage in St. Gallen dicselben nicht als irrige bezeichnet wurden), sondern aus der stellung der klöster St. Gallen und Weissenburg in den politischen wirren jener zeit und dem einflusse, den diese auf Grimalds lebensschicksale sicher, auf Otfrids wahrscheinlich austibten (vgl. Einl. s. 29 a. 38), wozu dann das schweigen der Weissenburger urkunden vortrefflich stimmt. Dass Otfrid Notkers persönliche bekanntschaft unmöglich schon 840 hat machén können, habe ich s. 38 ausdrticklich hervorgehoben. Zudem glaube ich nicht, dass Notker erst 840 geboren wurde. Der hauptbeweis, den Meyer von Knonau in den St. Galler Mittheilungen XV s. 4 dafür beibringt, ist hinfällig, denn hätte er wol (der brief an Liutbert ist 885 geschrieben; vgl. Dummler, Züricher Mitth. s. 259), von der im j. 862 erfolgten verwlustung des klosters Jumiẻges durch die Normannen sprechend, wo er also nach Meyer von Knonaus annabme bereits 22 jahre alt war, sagen können: cum adhuc iuuenculus essem! So spricht ein alter mann von einer längst vergangenen zeit, aber nicht ein funfundvierzigjähriger von einer zeit, dio erst ungefähr zwauzig jahre hinter ihm liegt. Es ist vielmehr die verwustung vom j. 841 gemeint, wie auch Diimmler a. a. o. annimmt, und Notker muss otwa 830 oder gar noch fruher geboren sein. Meyer von Knonaus librige erwägungen in der genannten anmerkung haben für ihu selbst nur accessorischen wert. - Ueber den von mir angenommenen dritten aufenthalt Otfrids in St. Gallen werde ich an andrem orte sprechen. Hier bemerke ich nur noch kurz, dass Meyer von Knonaus und meine vermutungen thber Lantaloh durchaus nicht unvereinbar sind.

$\mathrm{Zu}$ s. 31 meiner Einl. bemerke ich noch, dass man in bezug auf augaben der mönche uber das kloster, seine einrichtungen und persönlichkeiten nicht ausser augen lassen 
darf, dass es zum geistlichen stilus der zeit gehörte, in dieser hinsicht eine tibertriebene bescheidenheit zur schau zu tragen. So nennt noch Notker (D iammler, Zuricher antiquar. Mittheilungen XIII s. 219) St. Gallen einen paruus locus (vgl. dazu Diinmler a. a. o. s. 255).

\section{Noch einmal die handschriften.}

Ich hatte mir vorgenommen, die zweifellos irrige auffassung des neuesten bearbeiters eingehend zu widerlegen, damit es nicht hiesse: Qui tacet uidetur consentire. Allcin als ich mich daran machte, der confutatio durch eine apologia zu antworten, stellte sich heraus, dass die antwort auf die meisten punkte bereits in meiner confessio, der eiuleitung, enthalten ist und dass in einigen wenigen anderen sich meinung gegen meinung stcllt und es sich nur fragt, wer die seinige besser begründet. In beiden fällen zweifle ich nicht, dass meine auffassung des handschriftenverhältnisses vor einer nüchternen kritik stets billigung finden muss. So beschränke ich mich denn bier auf eine kurze würdigung der endergebnisse des neuesten bearbeiters. Es werden in der Wiener hds. 5 (6) schreiber angenommen. J. Haupt nimmt deren, wic or nir bei meiner jünsten anwesenheit in Wien (sommer 1880) sagte, noch minclestens zwei mehr an. Also die ansichten der gelchrten sind auch in diesen punkto noch nicht ubereinstimmend. Von zweien dieser schreiber sei hauptsächlich der text geschrieben, die sich besonders in der form des $\mathrm{g}$ unterschciden (denn dass die unterschiede in den formen des k, d, l, z, welche in zweiter reihe erwähnt werden, nicht festgehalten werden können, zeigen schon die beigegebnen facsimiletafeln, so ungenügend dieselben auch ausgefallen sind). Was nun die vermeintlichen orthographischen unterschiede beider schreiber angeht, so muss, wenn jemals thbertreibungen und gebrauch aller, auch der schwächsten waffen zeichen einer verlornen sache gewesen sind, dies von dem in bezug hierauf angefuhrten gelten. Dass die schreibung ii auch dem vermeintlichen ersten schreiber in $\mathrm{V}$ nicht unbekannt gewesen ist, zeigen die fälle IV, 2,33 (vgl. P. IV, 10, 12), III, 24, 40. 4, 3 (verglichen mit III, 19, 4; man könnte hier auch ii als ver- 
schriebenes u denken, doch das will ich meinem gegner nicht zu leide tun, denn sonst entgienge ihm auch eine seiner drei stellen, IV, 30, 16, die doch mit als unterscheidungsmerkmal des zweiten schreibers herbeigezogen ist). ${ }^{1}$ ) Das oh in IV, 26, 6. 18 gebe ich einfach $\mathrm{zu}$, doch begegnet dasselbe nicht ausser diesen beiden stellen noch 'oft', wio es dort heisst, sondern nur noch an zwci stellen (vgl. Einl. s. 124 no. 122), darunter an ciner gemeinsam mit $P$. Wer das allein aber als beweis eines andern schreibers gelten lassen will, der vertraut doch wahrlich einer schwachen stlitze. Auch das iiber die t-laute gesagte richtet sich selbst; man vergleiche nur die sammlungen bei Kelle II, $192 \mathrm{ff}$. und in meiner Einl. s. $110 \mathrm{f}$, , so wird man ausser den stellen des ziveiten schrcibers, auf welche die confutatio sich beschränkt, auch noch eine erkleckliche anzahl des ersten finden. Was endlich noch das 'viele andere', angeht, das 'jedem aufmerksamen beobachter von selbst auffallen muss', so habe ich es trotz aufmerksamer beobachtung vicht herausfinden können, muss also auf fernere enthüllungen warten. - Nun werden auch in der hds. $P$ wider zwei hauptschreiber angenommen, deren einer ein stück friiher einsetzt, als der entsprechende in $\mathrm{V}$ (was tibrigens ja zu der von mir s. 121 angenommenen reihenfolge in der successiven eutstehung vortrefflich stimmen würde). Merkwürdigerweise unterscheiden sich wider beide hauptsächlich in den formen des g. Nun hïtte ja der gedanke nahe gelegen, in dem ersten schreiber von $\mathrm{P}$ den ersten von $\mathrm{V}$, in dem zweiten von $\mathrm{P}$ den zweiten ron $\mathrm{V}$ wider $\mathrm{zu}$ erkennen. Allein abgeschen davon, dass dies ein bedenklicher erster schritt nach meiner ansicht hin gewesen wäre und dic hypothese fur sich doch viel unwahrscheinliches gehabt hätte, so wäro die folge eines solchen mangels an energic wahrscheinlich die gewesen, dass man die $k$ der ersten hand von $P$ vereinzelt auch in der zweiten hand ron V, das ziit des zweiten schreibers von V auch bei dem ersten schreiber von $\mathrm{P}$ widergefunden hätte und auch noch

1) Ich verkenne nicht, dass auch die von mir beigebrachten spuren zus dem ersten abschnitte dürftig sind, doch können sie sich jedenfalls an beweiskraft mit dem als hauptbeweismittel vorgeschobnen drei stellen wessen. 
viele andere ähnlichkeiten 1) herausgefunden, mit einem worte den zweiten schritt getan und erkannt hätte, dass der crste und zweite schreiber in $\mathrm{V}$ und der erste und zweite schreiber in $\mathbf{P}$ sammt und sonders eigentlich nur ein schreiber sind. Wie hilft sich nun der neueste bearbeiter aus der sich herausstellenden schwicrigkeit? Er nimmt (s. 19) zwei schreiberschulen an, von denen, wider merkwürdigerweise, in $\mathrm{P}$ und $\mathrm{V}$ die eine in dem ersten teil, die andere in dem zweiten hauptteile vertreten ist, er erkennt sogar an der beschaffenheit der hände, dass die beiden hdss. nicht allzulange nach einander geschrieben sind (vorsichtige kenner alter schrift schwanken oft um bundert jahre bei bestimmung einer hds. aus rein äusserlichen merkmalen), ja er komnt sogar zu der ansicht, dass die zweite schule wol die nusterschrift vertrete. Man erwäge das seltne zusammentreffen: zwei schreiberschulen, die beide in der form des $\mathrm{g}$ sich grimmig befehden, im $\mathrm{k}$ übergänge zeigen, im $\mathrm{z}$ unsicherheit, 'weil dasselbe für jeden an lateinischen texten geschulten schreiber eine ungewohnte aufgabe war', die aber sonst in ihren buchstabenformen und ligaturen, in ihren grammatischen, orthographischen, metrischen grundsätzen merkwärdige consequenz zeigen. Ich begreife nicht, dass er nicht vor dieser gewaltsamen lōsung selbst zurückwich. Die geringe verschiedenartigkeit der schrift und dinte in V., die ich selbst zugegeben habe, erklären sich höchst einfach dadurch, dass man bedenkt, dass in dem zeitraume von zwanzig jahren, in denen nach meiner annahme die hds. $V$ allmählich entstanden ist, doch auch die schrift des schreibers gewechselt haben muss. Wenn ich, was ich vor zwanzig jahren geschrieben, mit meiner heutigen schrift vergleiche, so finde ich, dass ich nicht nur civige buchstaben seit bestimnter zeit oder aus bestimmtem anlass, aus bequemlichkeitsgriunden u. dgl. anders schreile, sondern dass auch der allgemeine eindruck der schrift ein

1) Als solche hebe ich namentlich die eigentiimliche ligatur von th hervor, auf die mich J. Haupt für $\mathrm{V}$ aufmerksam machte und die ich ausserdew noch in $P$ (in beiden schreibern, ebenso von $V$ ), sonst aber nirgend gesehen zu haben mich erinnere. Dieselbe ist so gemacht (vgl. die facsimileblätter), dass der grundstrich des $t$ und der rechte haken des $h$ in einem zuge gemacht sind, der schaft des letzteren aber erst nachträglich hindurchgezogen ist. 
andrer ist, und wenn ich auch gern zugeben will, dass die schriftmalerei jener zeit mit der schriftschreiberei der heutigen nicht durchweg zu vergleichen ist, so verstehe ich doch nicht, warum selbst beim buchstabenmalen in solchem zeitlauf nicht änderungen denkbar sein sollen. Die grōssere gleichmässigkeit der schrift in $\mathrm{P}$, das seltnere vorkommen (nicht gänzliche meiden) der ubblichen ligaturen von st und rt, erklärt sich daraus, dass $P$ zum dedicationsexemplar bestimmt war. So sicher ich behaupte, dass $P$ den gereinigten, in unzähligen fällen verbesserten text darstellt, so wenig fällt mir ein zu leugnen, dass auch $P$ schreibfehler gemacht hat, vielleicht verhältnismässig mehr im hinblick auf seine vorlage $\mathrm{V}$, als $\mathrm{V}$ im hinblick auf seine vorlage $\mathrm{Kl}$; allein dabei ist zu bedenken, dass, wie ich Einl. s. 80 bemerkt habe, schreibfehler in einem schriftstlucke um so zablreicher sein müssen, je geringer in demselben die schaffende tïtigkeit des verfassers ist, dass also in den sogenannten reinschriften weit eher schreibfehler zu vermuten sind, als in den vielfach durchdachten ersten entwürfen. Daraus würde sich auch eine nachlässigkeit in der accentsctzung leicht crklären; allein es ist unrichtig, dass betonungen wie uuorólt, fírholan nur in $\mathrm{P}$ vorkommen; Einl. s. 157 habe ich ganz dieselbe erscheinung aus $\mathrm{V}$ nachgewiesen. Nun noch ein wort liber den unglücklichen corrector, der sich so vielerlei personalbestimmungen gefallen lassen muss. Man sollte doch bedenken, dass ein exemplar, in welchem corrigiert wird, keine reinschrift mehr ist, seitdem darin corrigiert wird, dass also die schrift der correcturen weit nachlässiger ausfallen muss, dass ferner das schreiben zwischen den zeilen eine gedrlickte, breitere form der schrift bedingt. Das wäre an sich begreiflich, bei Otfrid finden wir aber in den marginalien alle ubergänge von der schrift des correctors bis zu der saubersten schrift von $P$. Sogar die gemeinsame ibercorrectur leugnet die Berliner schrift, zu meinem grössten erstaunen. Wer correcturen, wie - ich greife ganz beliebige fälle heraus, die mir eben gegenwärtig sind - III, 7, 64 blyent; 14, 99 nemet; 22, 33 beginnet ir es; 15, 12 egypto; IV, 6, 6 guatu; 6, 37. 11, 5. V, 20, 8. 21, 9 und viele andere in V P selbst gesehen hat, oder wer bloss meine beschreibung derselben gelesen hat, kann nicht an der iden- 
tität des correctors zweifeln. Zahlreiche fälle s. Einl. s. $171 \mathrm{f}$. Wie werden nun diese correcturen erklärt? Die in $\mathrm{V}$ ausgefuhrtc correctur sei entweder anfangs ubersehen und erst bei der revision nachgetragen worden, oder sie wurde absichtlich so wie sie in $\mathrm{V}$ stand auch in $\mathrm{P}$ copiert! Nun stelle man sich einen mönch des 9. jahrh. mit einer solchen philologischen akribie vor, wenn man kann. Die sonstigen abschriften jener zeit zeigen wenige spuren davon, und selbst in unscrer zeit dürte ein so origineller kopf selten sein, der aus seiner vorlage nicht nur die correctur selbst aufnähme, sondern auch den schriftlichen vollzug derselben abmalte. Wem erscheint eine derartige deutung natürlich? Uebrigens glaube man nur nicht, dass etwa die eine correctur auf der tafel 5 die schreibweise der gewöhnlichen correcturen in $\mathrm{P}$ darstellte. Von diesen, welche genau denen in $\mathrm{V}$ gleichen, ist auf den beigegebuen tafeln leider nichts zu sehen.

Und nun erinnere ich noch kurz an die gewaltige zahl von übercinstimmungen äusserer und innerer art, die ich in beiden hdss. nacbgewiesen habe, um die erhobnen einwendungen in ihrer vollen diirftigkeit erkennen zu lassen. Ich behaupte, dass jeder sachverständige, der nur eine viertelstunde lang $\mathrm{P}$ und $\mathrm{V}$ nebeneinander vergleichen kann, uber ihr verhältnis nicht im unklaren bleiben wird.

Ich hätte noch manches zu sagen, allein da mir mein gegner ja demnächst gelegenheit zu ausführlicheren darlegungen geben wird, kann ich mich mit dem gesagten begntigen. Nur zwei punkte möchte ich hicr noch kurz berluhren, um bei der angedeuteton gelegenheit nicht pro domo sprechen zu mussen. Das erste ist, dass ich die in herrn dr. Erdmanns worten: Für die sprachliche erklärung der einzelnen stellen hat Piper die vorhandenen untersuchungen sorgfältig benutzt, nicht immer mit angabe der quelle (Z.f. d. Ph. XI, 124), liegende insinuation mit aller entschiedonheit zurlickweise, da ich den gewiss verzeiblichen grundsatz habe, derartige dinge nicht auf mir sitzen zu lassen, selbst auf die gefahr hin, durch die zurïckweisung erst die augen der fachgenossen darauf $\mathrm{zu}$ lenken.

Der zweite punkt betrifft die lageneinteilung in $\mathrm{V}$, für die in dankenswerter weise Scemüller das richtige bictet 
und bei der ich bekennen muss mich geirrt zu haben. Aber wer da weiss, wie argwöhnisch handgriffe, wie sic zur feststellung der quateruionencinteilung dienen, auf grossen bibliotheken beobachtet und wie energisch sie oft gehindert werden (dic stellung der quaternionummern ist bekauntlich nicht uberall dieselbe), der wird meinen irrtum entschuldbar finden. Ucbrigens ist der tatsäcbliche schaden nicht gross, da sich nahezu dieselben resultate aus der jetrigen sachlage ergeben, wie schon von anderer seite gezeigt worden ist.

AL'TONA, den 27. april 1881.

P. PIPER. 